Portland State University

PDXScholar

\title{
Structural Instability and Dynamic Emission Fluctuations in Zinc Oxide Random Lasers
}

\author{
Zachariah Peterson \\ Portland State University \\ Robert Campbell Word \\ Portland State University, wordr@pdx.edu \\ Rolf Könenkamp \\ Portland State University, rkoe@pdx.edu
}

Follow this and additional works at: https://pdxscholar.library.pdx.edu/phy_fac

Part of the Physics Commons

Let us know how access to this document benefits you.

\section{Citation Details}

Peterson, Z., Word, R. C., \& Könenkamp, R. (2018). Structural instability and dynamic emission fluctuations in zinc oxide random lasers. Journal of Applied Physics, 124(6), 063104.

This Article is brought to you for free and open access. It has been accepted for inclusion in Physics Faculty Publications and Presentations by an authorized administrator of PDXScholar. Please contact us if we can make this document more accessible: pdxscholar@pdx.edu. 


\title{
Structural instability and dynamic emission fluctuations in zinc oxide random lasers
}

\author{
Zachariah Peterson, R. C. Word, and R. Könenkamp \\ Physics Department Portland State University, Portland, Oregon 97201, USA
}

(Received 20 April 2018; accepted 26 July 2018; published online 10 August 2018)

\begin{abstract}
We report experimental results on the structural stability of optically pumped zinc oxide random lasers. We find that the lasing threshold is not entirely stable and depends on the accumulated light exposure received in pulsed optical pumping. We show that exposure levels below $\sim 1.5 \mathrm{~kJ} / \mathrm{cm}^{2}$ improve the lasing efficiency and lower the lasing threshold. Beyond that value, however, lasing efficiency and threshold begin to degrade. Electron microscopy shows that the degradation is accompanied by morphological changes characteristic of melting. These changes become visible at an exposure of $\sim 0.7 \mathrm{~kJ} / \mathrm{cm}^{2}$. We suggest that the melting is initially localized within nanometer areas and that the initial improvement is due to defect annealing. For exposures larger than $1.5 \mathrm{~kJ} / \mathrm{cm}^{2}$, the melting zones connect, leading to deterioration. The findings apply to coherent and incoherent lasing. If stable output from $\mathrm{ZnO}$ random lasers is desired, lower lasing thresholds or higher damage thresholds are needed. Published by AIP Publishing. https://doi.org/10.1063/1.5037108
\end{abstract}

\section{INTRODUCTION}

Zinc oxide with its direct bandgap at $3.37 \mathrm{eV}$ and an exciton binding energy of $\sim 60 \mathrm{meV}$ has found numerous photonic and electrical applications. ${ }^{1-3}$ Efficient excitonic emission from $\mathrm{ZnO}$ nanoparticles and comparably long exciton lifetimes make the material an interesting candidate for ultraviolet optically pumped random lasing. ${ }^{1,-13}$ Ease of fabrication, small mode volumes, and a variety of spectral characteristics lend themselves to three basic applications for $\mathrm{ZnO}$ random lasers: (A) The possibility of speckle-free lasing originating from a non-resonant amplification or the superposition of a large number of optically independent lasing events, (B) the possibility of generating unique spectral distributions from the combinations of just a few random lasing spots, and $(\mathrm{C})$ the possibility of preparing coherent nanoscale lasing sources in simple thin film arrangements. These applications utilize coherent and incoherent lasing regimes, and both of these regimes are accessible in random lasers. ${ }^{9}$ Coherent random lasing occurs when the light is sufficiently localized that amplification can occur in closed optical paths. For this to happen, strong scattering is needed with scattering lengths shorter than the wave vector. Resonance and coherence in this regime are then established for paths where the accumulated phase shift equals $2 \pi .{ }^{14}$ In the incoherent regime, the scattering length in the random medium is larger, and diffuse transport rather than localization prevails. In that case, closed paths have a low probability. While amplification can still be strong in this regime, the emission remains non-resonant and incoherent. When a large number of lasing modes with spectral overlap are excited in the same volume or in the same time frame, the emission linewidth is significantly broader than in the case where only a few single modes are observed. We address these regimes in the same $\mathrm{ZnO}$ material by using pump lasers with different excitation wavelengths and pulse durations. We find light-induced structural damage and optical property changes for these regimes. For the case of long excitation pulses, i.e., pulse durations of several nanoseconds, we find a very low degree of reproducibility in the emission spectra, similar to what has been observed by others. ${ }^{4,5,7,11-13}$ This stochastic behavior contrasts to the high reproducibility ${ }^{4-7}$ observed with picosecond excitation pulses. While one might suspect the stochastic behavior also to be caused by material damage, our results indicate that this is not the case. Instead, we agree with work that has attributed the low reproducibility to temporal fluctuations that occur when pump pulse times are greater than the exciton lifetime., ${ }^{4,5}, 13$ Our main emphasis, however, is on describing the extent and cause of a lightinduced instability in the operation of $\mathrm{ZnO}$ random lasers. We show that lasing thresholds and intensities depend sensitively on total accumulated light exposure. Exposure, as typically occurring during the pumping action, induces initially an enhancement of the lasing properties but then leads to an optical degradation. This degradation is correlated with structural changes that can be attributed to melting.

\section{EXPERIMENTAL DETAILS}

Polycrystalline $\mathrm{ZnO}$ nanoparticles of $240 \mathrm{~nm}$ average diameter were acquired from MKNano Inc. Thin nanoparticle films were prepared on Si or quartz substrates using particle suspensions in $95 \%$ ethanol. The substrates were cleaned by sonicating in $5 \mathrm{mM} \mathrm{HNO}_{3}$. The thin film preparation used a dip coating process with suspensions containing $20 \mathrm{~g} / \mathrm{L}$ $\mathrm{ZnO}$ nanoparticles. Typically, the dip-coating was repeated 20 times with a drying step at $\sim 100^{\circ} \mathrm{C}$ after each coating and ethanol rinsing after every five coatings. The samples were then annealed at $550^{\circ} \mathrm{C}$ in air for $1 \mathrm{~h}$.

For the lasing experiments, light pulses of $6 \mathrm{~ns}$ duration and a wavelength of $355 \mathrm{~nm}$ from the 3rd harmonic of a $\mathrm{Nd}$ :YAG laser were directed to the samples at $45^{\circ}$ incidence. Alternatively, $800 \mathrm{ps}$ light pulses at $337 \mathrm{~nm}$ from a $\mathrm{N}_{2}$ laser were used. An aperture and a quartz lens were used to define 
an illuminated area of $\sim 0.065 \mathrm{~mm}^{2}$. Emitted light from the sample was collected at an angle of $90^{\circ}$ from the surface plane using an optical microscope coupled to a fiber-optic spectrometer with a wavelength resolution of $0.72 \mathrm{~nm}$. Typical pulse energies for the nanosecond pulses were between 10 and $140 \mathrm{~mJ} / \mathrm{cm}^{2}$. Pulse energies for the picosecond pulses were between 2 and $40 \mathrm{~mJ} / \mathrm{cm}^{2}$. Both lasers were operated at a repetition rate of $\sim 1 \mathrm{~Hz}$.

Morphology changes were recorded using a FEI Sirion Scanning Electron Microscope (SEM) at an accelerating voltage of $15 \mathrm{kV}$. The light-exposed areas were marked, such that identical areas could be viewed before and after exposure to the pump lasers.

\section{RESULTS}

Figure 1 gives an overview on typical lasing spectra and their threshold behavior obtained in nano- and picosecond pumping. Part (a) of the figure shows a set of three single lasing emission lines on a broad photoluminescence background, as typically obtained in picosecond pumping. This type of spectrum is obtained when the pump light excites only a few lasing centers above threshold and when there is no spectral overlap among the different lasing spots. The single-line intensities over the photoluminescence background can be enhanced by adding optical enhancers, such as additional scatterers ${ }^{6,9}$ or plasmonic resonators, ${ }^{6}$ to the active material. The threshold behavior for the three lasing lines is shown in Fig. 1(b). Typical threshold fluences are of the order of a few $\mathrm{mJ} / \mathrm{cm}^{2}$.

Figure 1(c) shows cases of random lasing excited by nanosecond pump pulses. The line widths here are between $\sim 3$ and $8 \mathrm{~nm}$, i.e., markedly wider than for the single lines but significantly narrower than the width of the underlying photoluminescence spectrum which is around $15 \mathrm{~nm}$. The broader line width in this case is consistent with spectral overlap among a high density of lasing centers within the microscope field of view. For pump pulses with nanosecond duration, the lasing shows stochastic features, i.e., emission intensity, peak position, and linewidth vary significantly from shot to shot. Figure 1(d) shows this behavior in three spectra obtained from consecutive pump pulses with the same pump energy of $60 \mathrm{~mJ} / \mathrm{cm}^{2}$. Despite the stochastic behavior in nanosecond pumping, lasing thresholds for the incoherent lasing can reliably be determined from averaged spectra. The thresholds then present themselves as smooth increases of emission intensity vs. pump intensity with a corresponding linewidth reduction from $\sim 15 \mathrm{~nm}$ to $\sim 3 \mathrm{~nm}$. Figure 1(e) shows the behavior of emission intensity and linewidth for the threshold region for this case.

We next focus on the stability of the lasing under nanosecond pumping. The main results are shown in Fig. 2.

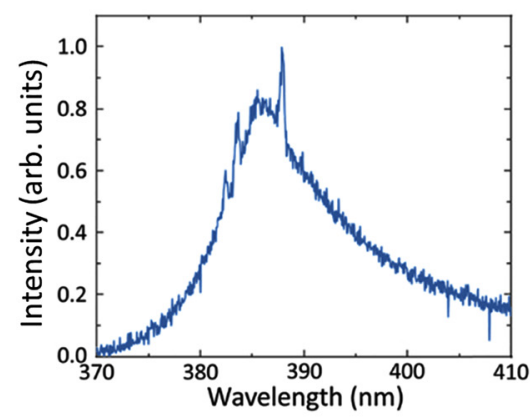

(a)

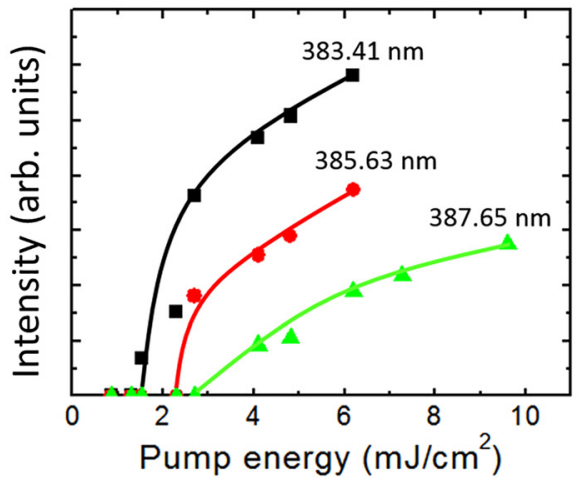

(b)

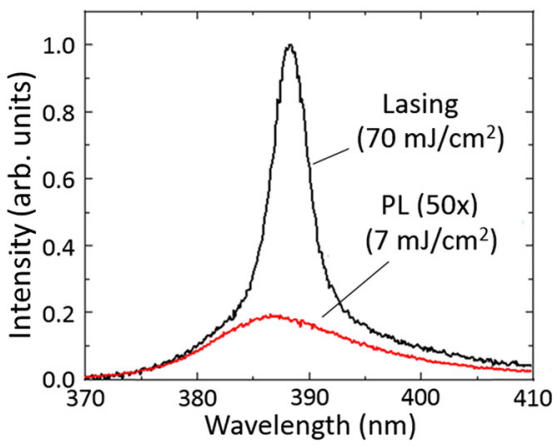

(c)

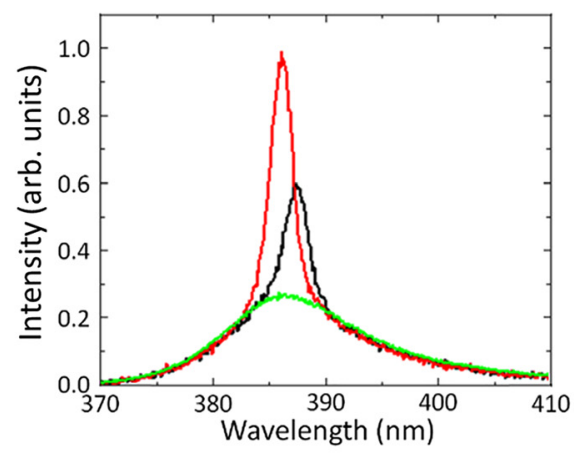

(d)

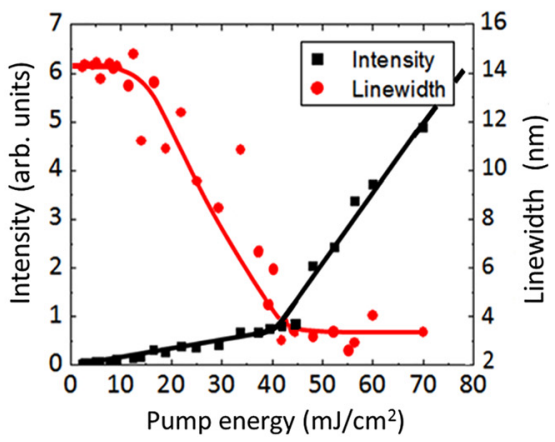

(e)

FIG. 1. (a) $\mathrm{ZnO}$ emission spectrum comprising single lasing lines obtained in the single-shot mode with $800 \mathrm{ps}$ excitations at $337 \mathrm{~nm}$ and a fluence of $7.3 \mathrm{~mJ} /$ $\mathrm{cm}^{2}$. (b) Threshold behavior of the three lasing lines visible in part (a). (c) Random lasing obtained with $6 \mathrm{~ns}$ pump pulses at $335 \mathrm{~nm}$ with fluences of $70 \mathrm{~mJ} /$ $\mathrm{cm}^{2}$ (above threshold) and $7 \mathrm{~mJ} / \mathrm{cm}^{2}$ (below threshold). (d) Three single-shot spectra obtained at the same pump pulse fluence of $60 \mathrm{~mJ} / \mathrm{cm}^{2}$, indicating stochastic fluctuations in the lasing peak location, intensity, and spectral distribution, obtained with nanosecond pump pulses. (e) Behavior of the intensity and linewidth in the threshold region. Linewidth narrowing is seen to precede the lasing threshold, and at threshold, a marked increase in the lasing emission is observed. The data points are obtained from averages. 


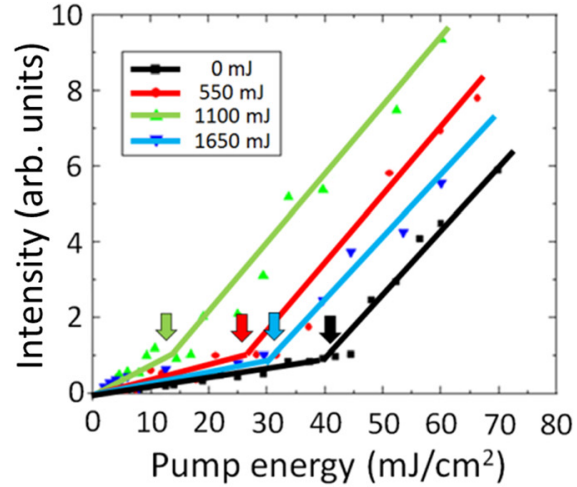

(a)

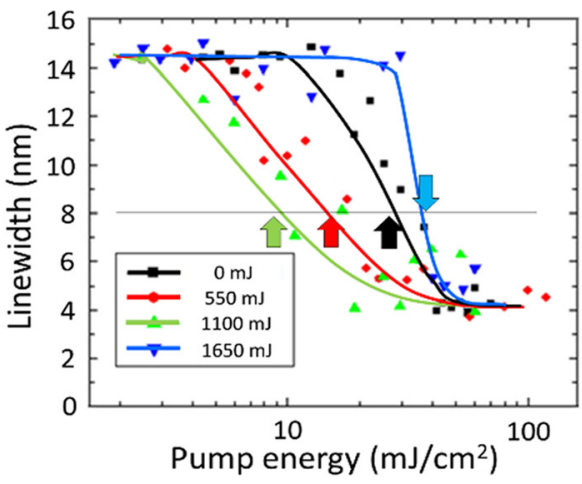

(b)

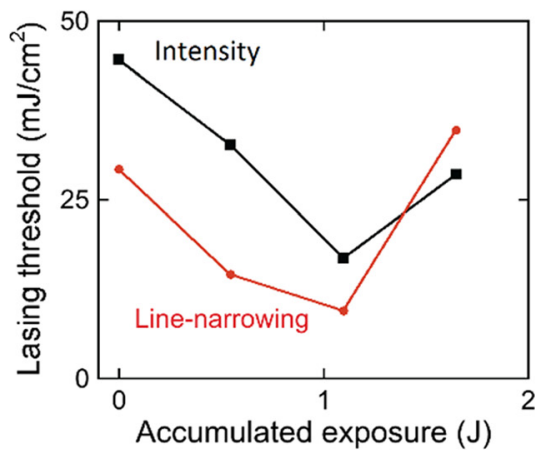

(c)

FIG. 2. (a) Emission intensity as a function of pump pulse energy for nanosecond pumping. The four curves were taken after a total light exposure as given in the legend. The lasing thresholds are marked by arrows. (b) Emission linewidth as a function of pump pulse energy after the sample received a total light exposure as indicated in the legend. The arrows indicate the linewidths slightly before the lasing threshold is reached. (c) Lasing threshold values as a function of total light exposure. Line-narrowing and intensity data from parts (a) and (b) of the figure were used.

Under repetitive pumping, it becomes apparent that the lasing intensities and the threshold values depend on the total accumulated light exposure received by the sample. The four threshold curves shown in Fig. 2(a) are related to total exposure doses between zero and $1 \mathrm{~J}$ for the exposed area of $0.065 \mathrm{~mm}^{2}$. The dose was administered in pulses with a fluence of $141 \mathrm{~mJ} / \mathrm{cm}^{2}$. To put these data into perspective, the exposure of $1 \mathrm{~J}$ corresponds to a fluence of $\sim 1.5 \mathrm{~kJ} / \mathrm{cm}^{2}$ and a sequence of $\sim 11000$ pulses at $140 \mathrm{~mJ} / \mathrm{cm}^{2}$. The figure reveals that for exposures up to this value, the light emission becomes stronger and the lasing thresholds decrease, corresponding to improved lasing properties. However, for exposure in excess of this value, a deterioration of the emission intensities and the lasing thresholds sets in corresponding to degrading lasing properties. A similar behavior is found for the spectral linewidth as shown in Fig. 2(b). The data show first a lowering of the linewidth, indicative of optical improvement, but for local exposures exceeding $1 \mathrm{~J}$, and a linewidth increase is observed-in this case to values well below the initial value. Figure 2(c) summarizes the data for the linewidth and the intensity in one plot.

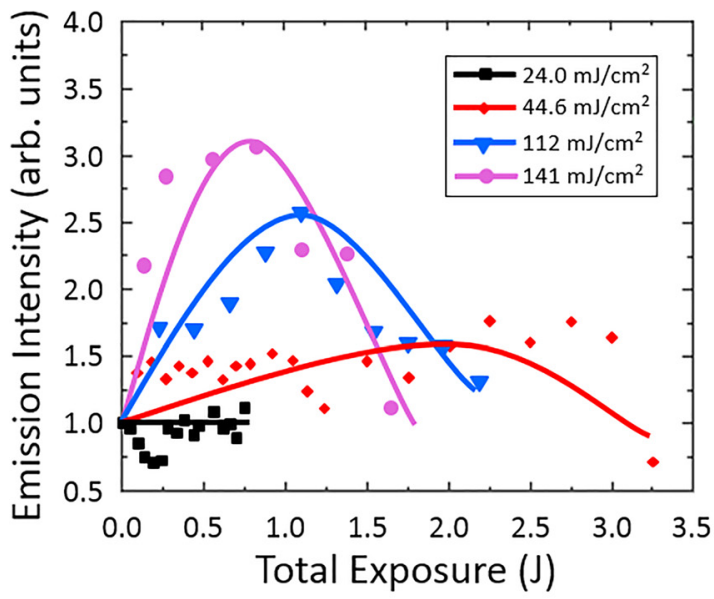

(a)
The material degradation depends sensitively on the pulse fluences used in the exposure, as shown in Fig. 3. While high pulse fluences induce strong changes already at comparatively low exposure doses, lower pulse fluences result in more moderate lasing changes and require larger doses to reach the maximum effect. When we take the initial slope for the curves in Fig. 3(a) as a measure of the material instability at a given pulse fluence, we can obtain an estimate of the stable lasing regime. This is done in Fig. 3(b) from which a damage threshold of approximately $20 \mathrm{~mJ} / \mathrm{cm}^{2}$ is extracted. This values lies within the band of typical lasing thresholds ranging from 10 to $40 \mathrm{~mJ} / \mathrm{cm}^{2}$, as shown in Fig. 2. The results thus indicate that stable operation of these lasers is not given.

We next turn to the morphological changes accompanying the optical changes. At low exposure levels, morphological changes cannot easily be detected. In particular, we were unable to resolve morphological changes after a single pulse exposure, even when the highest available pulse fluence of $141 \mathrm{~mJ} / \mathrm{cm}^{2}$ was applied. However, multi-pulse exposures generate material changes that can well be observed in the SEM. As shown in Fig. 4, the changes are initially confined

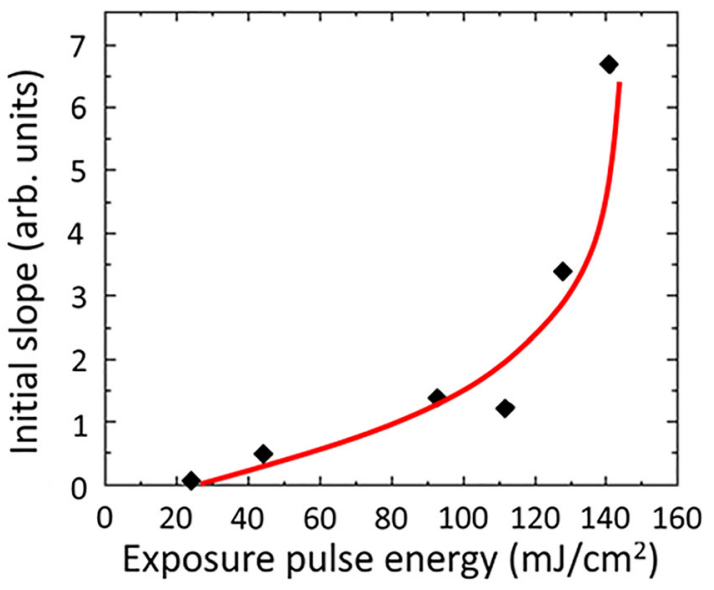

(b)

FIG. 3. (a) Evolution of incoherent lasing emission with increasing exposure to pump light of different pulse fluences. The data are qualitatively summarized by the solid curves. (b) Initial slopes for the curves in (a) versus the corresponding exposure pulse intensity. 


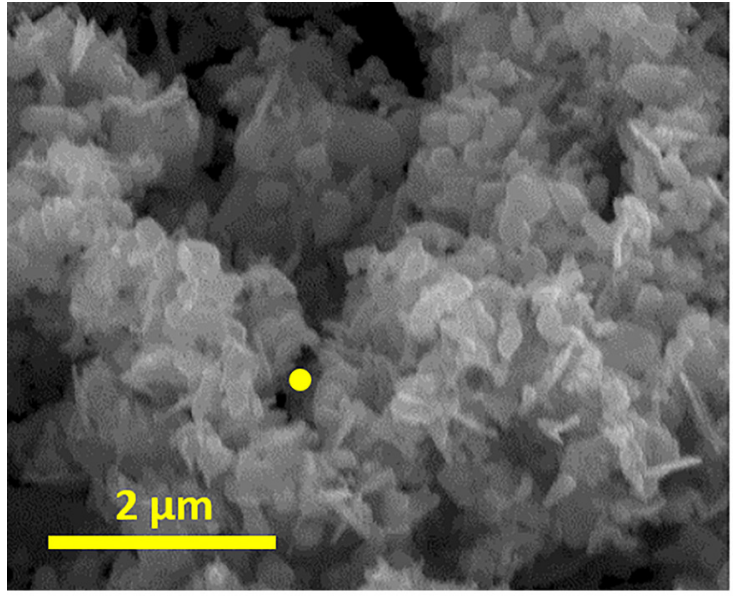

(a)

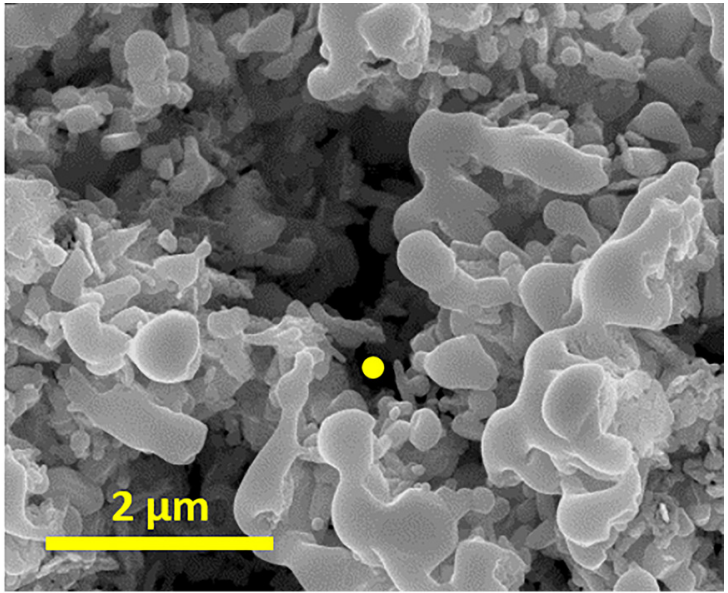

(b)

FIG. 4. (a) Scanning electron micrograph of $\mathrm{ZnO}$ nanoparticle films before light exposure. (b) The same area showing melting after an accumulated exposure of $550 \mathrm{~mJ}$ with a nanosecond pulse energy of $141 \mathrm{~mJ} / \mathrm{cm}^{2}$. Dots mark the same location in each image.

to areas of $\sim 100 \mathrm{~nm}$ at edges and corners of the crystallites but become extended and connected for accumulated exposures. Eventually, the exposed areas develop rounded, droplet-like surface regions indicative of melting. For an accumulated dose of $550 \mathrm{~mJ}$, clear morphological changes are discernible. While Fig. 4 was obtained at a fluence of $141 \mathrm{~mJ} / \mathrm{cm}^{2}$, the same effects are also seen at lower pulse fluence. For example, pulses of $60 \mathrm{~mJ} / \mathrm{cm}^{2}$ and a total exposure of $3.4 \mathrm{~J}$ produce melting, as shown in Fig. 5.

Finally, in Fig. 6, we show an example of induced melting with pulses in the picosecond regime. The findings are very similar to nanosecond pulse exposure. Melting is clearly observed for accumulated doses in excess of $1 \mathrm{~J}$ with a pulse energy of $9 \mathrm{~mJ} / \mathrm{cm}^{2}$ corresponding to 3-8 times the lasing threshold. It must hence be concluded that lasing excited with picosecond pulses suffers from damage in a similar way as lasing excited with nanosecond pulses. Only the damage thresholds are found to be somewhat larger relative to the lasing thresholds in this case.

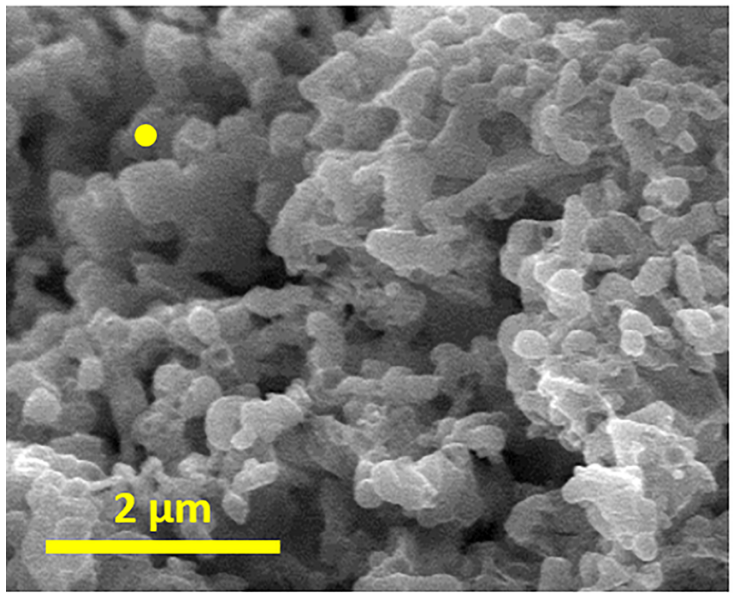

(a)

\section{DISCUSSION}

Several previous studies on $\mathrm{ZnO}$ nanoparticle films and structures indicate that annealing at moderate temperatures can lead to improvements in the optical properties. ${ }^{17,18} \mathrm{In}$ particular, improved luminescence, increases in the absorption coefficient near the absorption edge, and a steeper absorption tail have been reported. Later work indicated that pulsed laser annealing can lead to similar improvements, and transmission electron micrographs indicated that these changes are accompanied by relaxation of lattice strain and the removal of defects. ${ }^{15}$ We therefore attribute the initial improvements in lasing intensity and threshold, shown in Figs. 2 and 3, to laser-induced thermal annealing effects. An estimate for the temperatures induced in the pumping action can be obtained from the one-dimensional heat diffusion equation

$$
\rho c \frac{\partial T(x, t)}{\partial t}=k \frac{\partial^{2} T(x, t)}{\partial x^{2}}+\alpha I,
$$

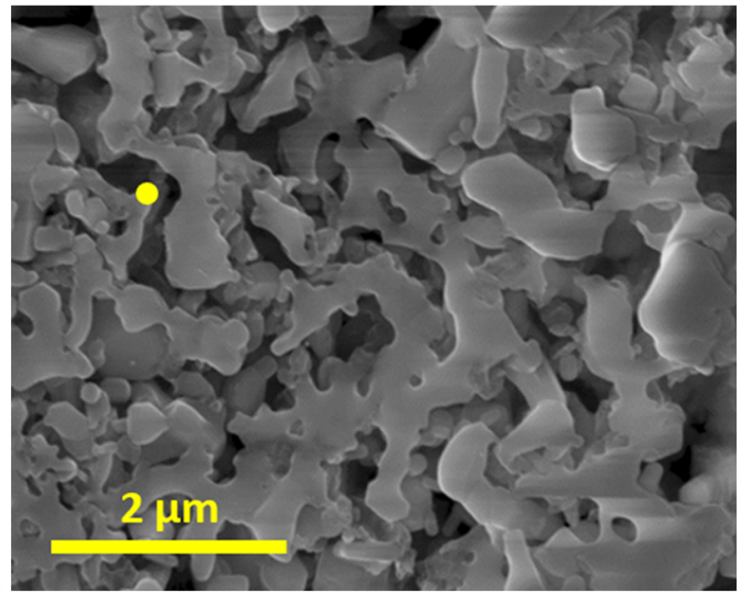

(b)

FIG. 5. (a) Film area before light exposure. (b) The same area as in (a) with melting visible after pumping with $60 \mathrm{~mJ} / \mathrm{cm}^{2}$ nanosecond pulses and a total exposure of $3400 \mathrm{~mJ}$. Dots mark the same location in each image. 


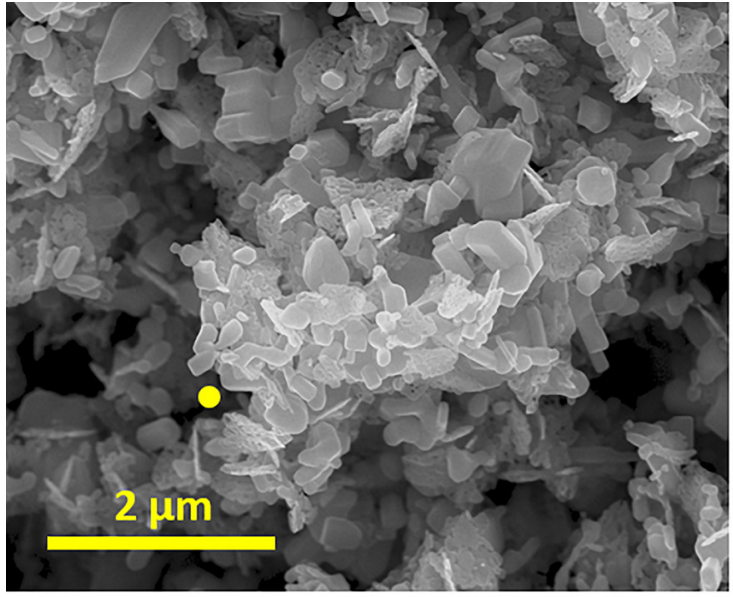

(a)

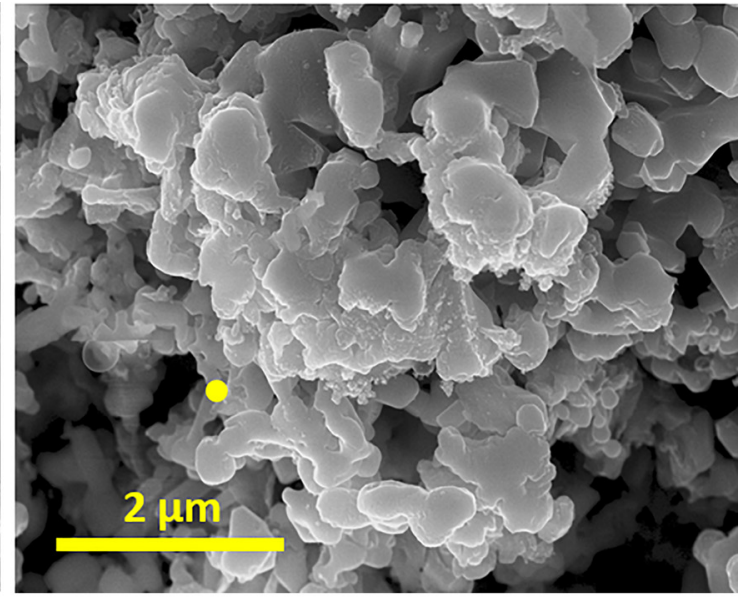

(b)

FIG. 6. (a) Sample area before light exposure. (b) The same area as in (a) after pumping with $9 \mathrm{~mJ} / \mathrm{cm}^{2}$ picosecond pulses and a total exposure of $990 \mathrm{~mJ}$.

where $T(x, t)$ is the temperature, $\rho$ is the density, $c$ is the specific heat, $k$ is the thermal conductivity, $\alpha$ is the absorption coefficient, and $I$ is the absorbed intensity. For a square pulse falling on to a semi-infinite film, an analytical solution for the temperature gain at the surface of the thin film and at the end of the pulse time $\tau$ is given by ${ }^{19}$

$$
\Delta T(0, \tau)=\frac{2 I_{0}(1-R)}{k}\left(\frac{D \tau}{\pi}\right)^{\frac{1}{2}}
$$

where $I_{0}$ is the laser intensity, $R$ is the reflectance, and $D$ is the thermal diffusivity. When we describe the light pulse as a constant flux $I_{O}$ with width $\tau, \Delta T(0, \tau)$ gives us an estimate of the temperature gain at the surface. Experimental data for chemically deposited $\mathrm{ZnO}$ nano-crystallite films reported by Ref. 20 were used for the thermal conductivity and diffusivity. It is noted that due to the loose packing of solution-deposited nano-particle films, these two parameters can be significantly lower than for compact thin films as obtained from evaporation, chemical vapor deposition, or sputtering. Using these data, we obtain a surface temperature of $2170 \mathrm{~K}$ for pump pulses of $6 \mathrm{~ns}$, a wavelength of $355 \mathrm{~nm}$, and a fluence of $\mathrm{I}_{0}=30 \mathrm{~mJ} / \mathrm{cm}^{2}$, and for pumping with pulses of $800 \mathrm{ps}$, a wavelength of $337 \mathrm{~nm}$, and a pulse fluence of $\mathrm{I}_{\mathrm{o}}=12 \mathrm{~mJ} / \mathrm{cm}^{2}$, we obtain $\mathrm{T}=2160 \mathrm{~K}$. These values are within a few per cent of the melting point for bulk $\mathrm{ZnO}$ of $2242 \mathrm{~K}$ and support our experimental observation of localized melting features in the exposed films, particularly when other contributing factors are considered, such as an inhomogeneous intensity distribution, non-linear absorption effects, and surface roughness. These effects would lead to an increased light absorbance during the laser pumping process, thereby favoring melting. Furthermore, it is well established ${ }^{21,22}$ that nano-structured films have somewhat reduced melting points and that the melting at nanoscale edges and vertices, as observed here, sets in at temperatures significantly lower than the melting point for the bulk material. Our estimates therefore make the localized melting a very plausible effect for the used operating conditions.
The degradation of the optical properties sets in at exposure levels that appear to coincide with the observation of melting. It therefore appears that stable device operation can be achieved only when the lasing thresholds are significantly decreased or if the damage thresholds are raised. Lower lasing thresholds have indeed been observed for $\mathrm{ZnO}$ random lasers prepared from homogenized spherical $\mathrm{ZnO}$ microparticles ${ }^{23,24}$ pointing towards a potential solution of the problem.

It was originally suggested in Refs. 11 and 12 that damage accumulation during individual pump pulses is also responsible for the spectral fluctuations observed in nanosecond optical pumping. However, damage effects could not be demonstrated. From the current work, it is clear that the damage produced by single laser pulses is very small and that only accumulated pulse exposure leads to significant optical changes. This contrasts quite clearly with the stochastic behavior in nanosecond pumping where spectral fluctuations of $\pm 30 \%$ between single pulses are observed in the lasing spectra and intensities, as shown in Fig. 1(d). It is thus difficult to explain the stochastic optical output with the comparably small damage induced by single pump pulses. Also, the observation of much higher reproducibility in picosecond pumping at pulse fluences well above the damage threshold indicates that damage is not the cause for stochastic lasing behavior. Alternative causes have been discussed in several publications ${ }^{4-7,11-13}$ which point to the shorter recombination lifetimes in the $\mathrm{ZnO}$ nanoparticles as compared to bulk $\mathrm{ZnO}$ : Essentially, when the recombination lifetimes are substantially shorter than pump pulse times, temporal sequences of un-coupled lasing events can occur within a single pump pulse, and the dynamic evolution of these sequential lasing events is suggested to produce the large shot-to-shot variations in nanosecond pumping.

\section{CONCLUSIONS}

We have presented experimental results, indicating a structural instability in $\mathrm{ZnO}$ random lasers induced by optical pulses in the picosecond and nanosecond range. We have 
identified damage thresholds for $\mathrm{ZnO}$ particle films that are in the same range as the lasing thresholds. For the wavelengths and pulse lengths selected here, the damage thresholds for random lasing are somewhat higher than the lasing thresholds, and the operational stability is hence somewhat better. We find the optical properties first to improve but subsequently to deteriorate. The initial improvement may be attributed to annealing effects, ${ }^{14,16,17}$ while the deterioration is associated with accumulating melting. At pump fluences near and above the lasing thresholds, melting behavior is observed. Estimates of the surface temperature reached in the pumping process are within a few percent of the $\mathrm{ZnO}$ melting point. These results clearly indicate that stable random lasing in this type of film will remain a challenge.

As others before us, ${ }^{4,5,7,11-13}$ we find strong spectral fluctuations in the lasing output when nanosecond pulses are used for pumping, while picosecond pumping generates very reproducible lasing spectra. From the results presented here, it appears unlikely that the shot-to-shot spectral fluctuations in nanosecond pumping are caused by structural damage.

${ }^{1}$ D. M. Bagnall, Y. F. Chen, Z. Zhu, and T. Yao, "Optically pumped lasing of ZnO at room temperature,” Appl. Phys. Lett. 70, 2230 (1997).

${ }^{2}$ A. Nadarajah, R. C. Word, J. Meiss, and R. Könenkamp, "Flexible inorganic nanowire light-emitting diode," Nano Lett. 8, 534 (2008).

${ }^{3}$ A. Nadarajah, R. C. Word, K. VanSant, and R. Könenkamp, "Nanowire-quantum-dot-polymer solar cell," Phys. Status Solidi B 245, 1834 (2008).

${ }^{4}$ V. M. Markushev, M. V. Ryzhkov, and C. M. Briskina, "Characteristic properties of $\mathrm{ZnO}$ random lasers pumped by nanosecond pulses," Appl. Phys. B 84, 333 (2006).

${ }^{5}$ V. M. Markushev, M. V. Ryzhkov, C. M. Briskina, and H. Cao, "Spectra of $\mathrm{ZnO}$ random lasers under nanosecond pumping," Proc. SPIE 6320, 63200B (2006).

${ }^{6} \mathrm{D}$. Wiersma, "The physics and applications of random lasers," Nat. Phys. 4, 359 (2008).

${ }^{7}$ J. Fallert, R. J. B. Dietz, M. Hauser, F. Stelzl, C. Klingshirn, and H. Kalt, "Random lasing in ZnO nanocrystals," J. Lumin. 129, 1685 (2009).
${ }^{8}$ H. Cao, "Random lasers with coherent feedback," Optical Properties of Nanostructured Random Media, Topics in applied physics (Springer, 2002), Vol. 82, p. 303.

${ }^{9}$ H. Cao, J. Y. Xu, S. H. Chang, and S. T. Ho, "Transition from amplified spontaneous emission to laser action in strongly scattering media," Phys. Rev. E 61, 1985 (2000).

${ }^{10}$ J. Fallert, R. J. B. Dietz, J. Sartor, D. Schneider, C. Klingshirn, and H. Kalt, "Co-existence of strongly and weakly localized random laser modes," Nat. Photonics 3, 279 (2009).

${ }^{11}$ Y. Sun, J. B. Ketterson, and G. K. L. Wong, "Excitonic gain and stimulated ultraviolet emission in nanocrystalline zinc-oxide powder," Appl. Phys. Lett. 77, 2232 (2000).

${ }^{12}$ N. T. Binh, P. V. Thin, P. T. Nga, and N. A. Tuan, "Random lasing from a layer of $\mathrm{ZnO}$ powder painted on glass substrate under excitation of nanosecond and picosecond pulses," Commun. Phys. 21, 353 (2011).

${ }^{13}$ V. M. Markushev, M. V. Ryzhkov, C. M. Briskina, H. Cao, H. Zhong, S. W. Wang, and W. Lu, "Analysis of $\mathrm{ZnO}$ random laser spectra under nanosecond pumping," Proc. SPIE 6731, 67311M (2007).

${ }^{14}$ H. Cao, Y. G. Zhao, S. T. Ho, E. W. Seelig, Q. H. Wang, and R. P. H. Chang, "Random laser action in semiconductor powder," Phys. Rev. Lett. 82, 2278 (1999).

${ }^{15}$ A. Nadarajah and R. Könenkamp, "Laser annealing of photoluminescent $\mathrm{ZnO}$ nanorods grown at low temperature," Nanotechnology 22, 025205 (2011).

${ }^{16}$ R. Niyuki, H. Fujiwara, Y. Ishikawa, N. Koshizaki, T. Tsuji, and K. Sasaki, "Toward single-mode random lasing within a submicrometre-sized spherical ZnO particle film,” J. Opt. 18, 035202 (2016).

${ }^{17}$ S. Dutta, S. Chattopadhyay, and D. Jana, "Annealing effect on nano-ZnO powder studied from positron lifetime and optical absorption spectroscopy,” J. Appl. Phys. 100, 114328 (2006).

${ }^{18} \mathrm{~V}$. Noack and A. Eychmüller, "Annealing of nanometer-sized zinc oxide particles," Chem. Mater. 14, 1411 (2002).

${ }^{19}$ J. H. Bechtel, "Heating of solid targets with laser pulses," J. Appl. Phys. 46, 1585 (1975).

${ }^{20} \mathrm{~K}$. T. Igamberdiev et al., "Thermal properties of semiconducting zinc oxide nanostructures," J. Eng. Phys. Thermophys. 83, 863 (2010).

${ }^{21}$ G. Guisbiers and S. Pereira, "Theoretical investigation of size and shape effects on the melting temperature of $\mathrm{ZnO}$ nanostructures," Nanotechnology 18, 435710 (2007).

${ }^{22}$ Z. Zhang, M. Zhao, and Q. Jiang, "Melting temperatures of semiconductor nanocrystals in the mesoscopic size range," Semicond. Sci. Technol. 16, L33 (2001).

${ }^{23}$ T. Nakamura, S. Sonoda, T. Yamamoto, and S. Adachi, "Discrete-mode ZnO microparticle random laser," Opt. Lett. 40, 2661 (2015).

${ }^{24} \mathrm{H}$. Fujiwara et al., "Low threshold and quasi-single mode random laser within a submicrometer-sized $\mathrm{ZnO}$ spherical particle film," Appl. Phys. Lett. 102, 061110 (2013). 